\title{
A GRADIENT FLOW FORMULATION FOR THE STOCHASTIC AMARI NEURAL FIELD MODEL
}

\author{
CHRISTIAN KUEHN AND JONAS M. TÖLLE
}

\begin{abstract}
We study stochastic Amari-type neural field equations, which are mean-field models for neural activity in the cortex. We prove that under certain assumptions on the coupling kernel, the neural field model can be viewed as a gradient flow in a nonlocal Hilbert space. This makes all gradient flow methods available for the analysis, which could previously not be used, as it was not known, whether a rigorous gradient flow formulation exists. We show that the equation is well-posed in the nonlocal Hilbert space in the sense that solutions starting in this space also remain in it for all times and space-time regularity results hold for the case of spatially correlated noise. Uniqueness of invariant measures, ergodic properties for the associated Feller semigroups, and several examples of kernels are also discussed.
\end{abstract}

\section{INTRODUCTION}

The stochastic integro-differential equation we study is

$$
\partial_{t} u=-\alpha u+\int_{\mathcal{B}} w(\cdot, y) f(u(y, t)) \mathrm{d} y+\varepsilon \partial_{t} W
$$

where $(x, t) \in \mathcal{B} \times[0, T), \mathcal{B} \subset \mathbb{R}^{d}$ is the spatial domain modeling the cortex, $u=u(x, t) \in \mathbb{R}$ is the neural field interpreted as the average/mean-field activity of the neural network, the kernel $w$ models the connections between neurons, $f$ is the main nonlinearity representing the firing rate, $\alpha>0$ is the decay rate, and $W=W(x, t)$ is a (spatially correlated, additive) noise controlled via the parameter $\varepsilon \geq 0$. We shall write $B W$ instead of $W$ below, where $B$ is the covariance operator. The precise mathematical formulation for (1.1) starts in the subsequent Section 2, In this introduction, we outline the main setting and our results.

Deterministic neural field models are well-established in the modeling of mean-field cortex activity. The version (1.1) (for $\varepsilon=0$ ) is frequently attributed to Amari 2] while a version with $f$ outside the integral is attributed to Wilson and Cowan [73]. Since the kernel $w$ models neuronal synaptic connections, it is often parametrized to ensure that different connection structures can be modeled, which may arise e.g. due to synaptic plasticity. The noise term may either be viewed as modeling external input noise or it can be viewed as a correction due to finite population size or population heterogeneity.

A key motivation to study neural fields arises from neurological disorders, such as Parkinson or epilepsy. For example, epileptic seizures can be tracked by recording EEG (or ECoG) data, which essentially represent a partial neural field measurement on the upper (or lower) layers of the cortex. The general hypothesis is that there are temporary phases, also called epileptic

Date: November 27, 2018.

2010 Mathematics Subject Classification. Primary: 60H15; 92C20; Secondary: 34B10; 35B65;

Key words and phrases. Gradient flow in Hilbert space; stochastic Amari neural field equation; nonlocal Hilbert space; spatially correlated additive noise; space-time regularity of solutions; nonnegative kernel; unique invariant measure; ergodic Feller semigroup; mathematical neuroscience.

CK acknowledges financial support by a Lichtenberg Professorship. JMT would like to thank Dirk Blömker for some useful comments. Both authors are indebted to the anonymous reviewers for several helpful remarks. 
seizures, during which the brain operates in a completely different dynamical regime. Whether this changing activity pattern can be related/modeled to changing the parameters in the neural connectivity network, and therefore changing the kernel in a neural field model, remains an open problem currently under discussion in multiple scientific communities.

However, even during normal functioning of the brain, several topics such as wave propagation, visual hallucinations, orientation and memory functions have been connected to continuum neural field models [13] such as (1.1); see also [16, 24] for broader introductions and further references. Although the analysis of spatio-temporal pattern-formation for deterministic neural fields is well-established, only very recently there has been a surging interest in stochastic neural field equations. One broad motivation is to understand the effect of finite population size [12, 60, 68], while another is to take into account environmental fluctuations. A very concrete application is to understand the role of noise-induced fluctuations [63, 72] in perceptual bistability [54, 70]. More precisely, it has been hypothesized that noise-induced bistability in neural fields switching between different invariant sets is related to switches between visual perception of ambiguous visual patterns. An example that noise-induced switching between locally stable deterministic steady states is indeed possible for (1.1) is shown in Figure 1.1
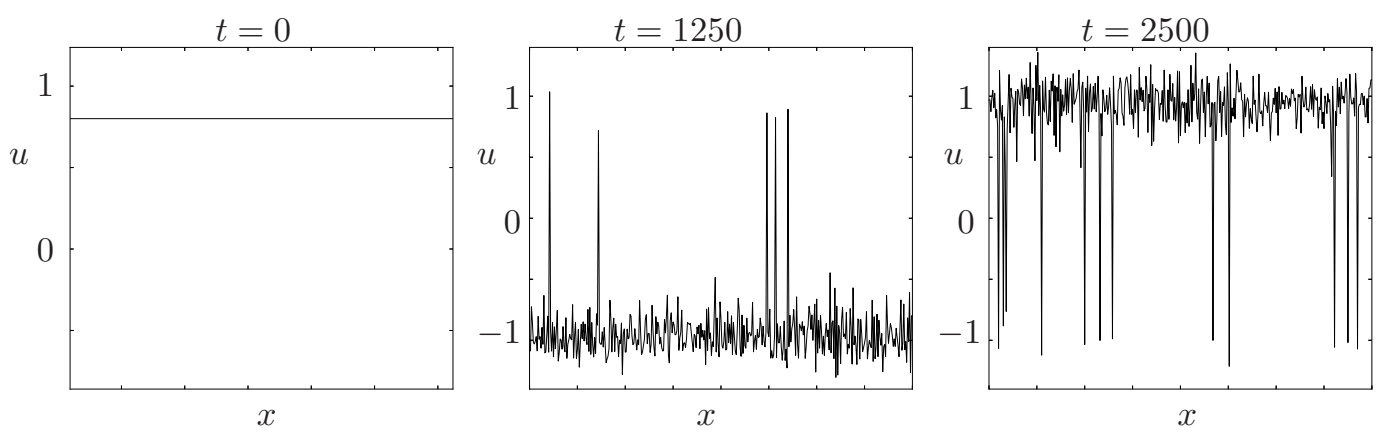

Figure 1.1. Numerical simulation (1.1) with $f(u)=(u+1)(1-u)(u-0.1)$, $w(x, y)=J(x-y)$ for $J(z)=1 /(\tilde{\sigma} \sqrt{2 \pi}) \exp \left(-z^{2} /(2 \tilde{\sigma})\right)$ for $\tilde{\sigma}=0.05, x \in \mathcal{B}=$ $[-80,80], \alpha=0.1$, space-time white noise with $\varepsilon=0.5$, and $u(x, 0) \equiv 0.8$. The three snapshots clearly show that there is metastability between two locally deterministically stable states. In particular, the neural field behaves reminiscent of a gradient-type system with additive noise.

In summary, the main biological insights to be gained from continuum neural field models are obtained via the analysis of the patterns they produce, and how these patterns depend upon parameters. A general observation for many different choices of kernels $w$ and firing rates $f$ is that multiple patterns are possible, while for certain parameter ranges the model seems to select just a single pattern. To clarify this effect, one natural conjecture is that one should use mathematical analysis to determine, whether there are conditions, e.g. on the kernel $w$ that lead to different mathematical evolution equation structures, which naturally distinguish between a neural field operating in a multistable pattern formation mode, or in a single global dissipative mode.

The basic theory for (1.1), including existence, regularity, Galerkin approximations and large deviations of (1.1), has been covered in the work by Kuehn and Riedler 44] for bounded domains, while unbounded domains are considered by Faugeras and Inglis [28]. The influence of noise on traveling waves in stochastic neural fields has been studied intensively in recent years [15, 37, 40, 42, 43, 47, 57]. Yet, a sharp study of noise-induced switching rates can only be carried out for (1.1) beyond standard large deviations if one can use a gradient structure, which is 
required, e.g., to generalize Kramers' law 8]. In fact, trying to transfer the Kramers' law results from the local stochastic partial differential equation setting in [8] to the nonlocal stochastic integral equation (1.1) was one of the motivations for this work; see also Figure 1.1 for the numerical confirmation that gradient-like noise-induced switching dynamics can appear in neural field models.

However, even in the deterministic setting $(\varepsilon=0)$ it is not known, whether we can find a gradient structure to rewrite (1.1) as

$$
\partial_{t} u=-\nabla_{\mathcal{X}} \mathcal{F}(u), \quad u(\cdot, t)=u(t) \in \mathcal{X},
$$

where $\mathcal{X}$ is a suitable function space, e.g., a Hilbert, Banach or even metric space [3] , and $\mathcal{F}: \mathcal{X} \rightarrow \mathbb{R}$ is a functional, which often has the natural interpretation of an energy, entropy, or some other physical notion. Of course, to have such a gradient structure in the (stochastic) neural field case would not only be interesting for Kramers' law as suggested by Kuehn and Riedler [44] but also open up a general area of techniques, which has been extremely successful for other differential equations [38, 56].

Furthermore, if we can characterize the types of kernels for which gradient structures exist, it would give us an understanding, if and when the brain might be working in two different regimes such as energy-decay versus complex non-equilibrium pattern formation. Unfortunately, direct calculations in several works have shown [23, 44] that one does not expect any gradient-structure of the form (1.2) in the standard Lebesgue and Sobolev spaces for neural fields, despite many analogies to certain classical PDEs with such structures [46]. See [22] for a recent contribution on the topic of gradient flows for neural field equations with weights in spaces of continuous functions.

In this work, we show that a gradient structure actually does exist for (stochastic) neural fields. We construct a nonlocal Hilbert space $\mathcal{X}$ using functional-analytic methods, which allows us to obtain an exact gradient system. For the gradient framework, we analyze well-posedness of the resulting system in the nonlocal space $\mathcal{X}$ using methods from stochastic analysis. Using the gradient structure we can characterize invariant measures of the process. Furthermore, we investigate ergodic properties of Feller semigroups generated by the neural field equation. We note that a related idea was used for the stochastic porous media equation using monotonicity methods by Ren et al. [59], Röckner and Wang [61].

Furthermore, we crucially note that $\mathcal{X}$, and the related gradient structure, only exist for a certain classes of kernels with sufficiently dominant excitation or inhibition (see Section 4 below for examples), while it fails for other classes. Kernels may, depending upon parameters, fall into different classes so that in underlying applications we might obtain major transitions in neural fields between different mathematical structures. In fact, a similar transition between classes has recently been observed in the context of entropy/gradient-structures for local cross-diffusion systems [39] modeling herding behavior, where parameter variation can destroy the entropy structure.

We conjecture that our new approach may allow us to now consider many other stochastic variants of models from biological applications, e.g., nonlocal Fisher-KPP equations [1, 7, 33], nonlocal aggregation models [67], or nonlocal swarming systems [53] via a gradient flow approach. Another important future perspective of our framework is to obtain sharp asymptotics for nonlocal small noise asymptotics and large deviation principles [21, Chapter 12] similar to Kramers' law recently obtained for stochastic partial differential equations [6, 9, 10].

Structure of the the paper. In Section 2 we introduce the mathematical model for equation (1.1) and give the main Assumptions 13 for the existence and uniqueness of solutions to the stochastic evolution equation (2.1). In Subsection 2.1. we shall introduce the nonlocal Hilbert space and the gradient flow structure on it. The stochastic gradient flow is introduced in Subsection 
2.2. where also our final Assumption 4 is formulated. Invariance and pathwise regularity of the solutions are discussed in Subsections 2.3 and 2.4 respectively. The existence and uniqueness of invariant measures and the ergodicity of the semigroup associated to the stochastic equation are studied in Section 3 In Section 4 we give examples for the nonnegativity condition of the kernels and discuss an equivalent condition for this which is given by Fourier transforms. The concluding summary is given in Section [5, followed by Appendix $\mathbf{A}$ on cylindrical Wiener processes.

\section{The Stochastic Amari Model}

Let $\mathcal{B} \subset \mathbb{R}^{d}$ be non-empty, bounded and closed. From now on, denote

$$
H:=L^{2}(\mathcal{B}) \text {. }
$$

Formalizing equation (1.1), let $\alpha>0$ (decay rate for the potential), $\varepsilon \geq 0$ (noise level parameter) and consider the following stochastic integro-differential equation in $H$ :

$$
\mathrm{d} U_{t}=\left[-\alpha U_{t}+K F\left(U_{t}\right)\right] \mathrm{d} t+\varepsilon B \mathrm{~d} W_{t}, \quad U_{0}=u_{0} \in L^{2}(\mathcal{B}), \quad t \in[0, T],
$$

where $T>0$ is some finite time horizon. Let us discuss the assumptions on the coefficients.

Assumption 1. Let $f: \mathbb{R} \rightarrow(0,+\infty)$ be a globally Lipschitz continuous gain function.

Typically, we might want to include the sigmoid function

$$
f(s)=\left(1+\mathrm{e}^{-s}\right)^{-1} \quad \text { or } \quad f(s)=(\tanh (s)+1) / 2
$$

as examples, which are commonly used in the analysis of neural fields [13]. Let $F(g)(x):=$ $f(g(x)), g \in H$, be the associated Nemytskii operator for $f$. Since $f$ is Lipschitz, we get that $F$ is a nonlinear Lipschitz continuous operator $F: H \rightarrow H$, see e.g. [64, Theorem II.3.2].

Let

$$
K g(x):=\int_{\mathcal{B}} J(x-y) g(y) \mathrm{d} y, \quad g \in L^{2}(\mathcal{B}),
$$

where $J: \mathbb{R}^{d} \rightarrow \mathbb{R}$, measurable, satisfies the following.

Assumption 2. Assume that

(i) $J(x)=J(-x)$, for every $x \in \mathcal{B}+\mathcal{B}:=\{x+y: x, y \in \mathcal{B}\}$,

(ii) $J \in L^{2}(\mathcal{B}+\mathcal{B}) \cap C(\mathcal{B}+\mathcal{B})$,

(iii) $J$ gives rise to a nonnegative definite kernel in the following sense

$$
\sum_{i, j=1}^{n} c_{i} c_{j} J\left(x_{i}-x_{j}\right) \geq 0
$$

for any choice of $n \in \mathbb{N},\left\{x_{1}, \ldots, x_{n}\right\} \subset \mathcal{B}$, and $\left\{c_{1}, \ldots, c_{n}\right\} \subset \mathbb{R}$.

This assumption that the kernel $w$ in (1.1) only depends on the difference is typical for neural field models [13]. Obviously, by (i), (ii) above, $K$ is a self-adjoint Hilbert-Schmidt operator 1 on $H$ that is, $K \in L_{2}(H)$. By a generalization of Mercer's theorem to compact spaces, $K$ is even of trace class, that is, a nuclear operator, cf. [31, Theorem 2.6 and the remark thereafter]. Denote by $\sigma(K)=\mathbb{C} \backslash \rho(K)$ the spectrum of $K$, where $\rho(K)$ is the resolvent set, see e.g. [58]. By (iii) above and by [29, Theorem 2.1] $K$ is nonnegative definite (as a linear operator on $H$ ), that is,

$$
\int_{\mathcal{B}} f(x) K g(x) \mathrm{d} x \geq 0, \quad f, g \in L^{2}(\mathcal{B}),
$$

see also [30, Theorem 3.1]. See Section 4 below for examples of kernels that satisfy (2.2). In Section 4 we shall discuss also particular examples of kernels and models, among others, from

\footnotetext{
${ }^{1}$ Let $U, V$ be separable Hilbert spaces, $L_{2}(V):=L_{2}(V, V)$, where $L_{2}(U, V)$ denotes the space of Hilbert-Schmidt operators from $U$ to $V$.
} 
works of Bressloff [14], Coombes et al. 17], Ermentrout et al. 25], Laing [45], Veltz and Faugeras 71].

Furthermore, on a filtered normal probability space $\left(\Omega, \mathcal{F},\left\{\mathcal{F}_{t}\right\}_{t \geq 0}, \mathbb{P}\right)$, let $\left\{W_{t}\right\}_{t \geq 0}$ be a cylindrical Wiener process with values in $H$ and covariance $Q:=$ Id equal to the identity operator on $H$, see Appendix $₫$ for details. Now, let $B \in L(H)$ denote the coefficient/covariance operator for the noise term in our equation.

Assumption 3. Assume that the covariance operator of the noise $B \in L_{2}(H)$ is Hilbert-Schmidt, nonnegative and symmetric.

This assumption essentially enforces the need for spatial correlations in dimension $d \geq 2$, see 21]. However, these correlations are indeed quite natural in the modeling setup so we do not give up relevant generality here. See e.g. [13, p. 91, equation (6.55)] or [14, Equation (9.44)] for a justification in terms of neuroscience to consider a continuum limit model for spatial correlations of the noise.

Now, we have access to stochastic integration theory in infinite dimensions, in particular, the expressions

$$
B W_{t}=\int_{0}^{t} B \mathrm{~d} W_{s}, \quad \text { and } \quad\left(v, B W_{t}\right)_{H}, v \in H, t \geq 0,
$$

for the cylindrical Wiener process $\left\{W_{t}\right\}_{t \geq 0}$ as above can be given a meaning, see [21, Proposition 4.26 and its proof]. Note that by our assumptions, $\left\{B W_{t}\right\}_{t \geq 0}$ is just a $B^{2}$-Wiener process on $H$. However, we choose to make use of the more complicated formalism of cylindrical Wiener

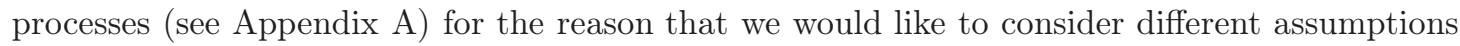
on regularity for $B$ (see e.g. Assumption 4 below) so that $B$ helps to keep track of the spatial correlations in the notation.

We continue by recalling the main solution concept.

Definition 2.1. A solution to (2.1) is a predictable stochastic process $U \in L^{2}(\Omega \times[0, T] ; H)$ with $\mathbb{P}$-a.s. Bochner integrable trajectories $t \mapsto U_{t}$ and with $U_{0}=u_{0} \in H=L^{2}(\mathcal{B})$ such that

$$
\left(U_{t}, v\right)_{H}=\left(u_{0}, v\right)_{H}+\int_{0}^{t}\left(-\alpha U_{s}+K F\left(U_{s}\right), v\right)_{H} \mathrm{~d} s+\varepsilon\left(v, B W_{t}\right)_{H},
$$

for every $v \in H, t \in[0, T], \mathbb{P}$-a.s.

Lemma 2.2. Under our assumptions, for $u_{0} \in H$, a mild solution in the sense of [21, Chapter 7] to (2.1) is a solution to (2.1) and vice versa.

Proof. We note that $F: H \rightarrow H$ is a bounded operator and that the domain of the infinitesimal generator of the $C_{0}$-semigroup $\left\{e^{-t \alpha}\right\}_{t>0}$ is all of $H$ (which is just identity times $-\alpha$ ). The claim follows now from [21, Theorem 5.4 (and its proof) and Appendix A]. See also [48, Proposition G.0.5].

Remark 2.3. Note that in our case, the notion of a solution in $H$ coincides both with the socalled analytically strong solutions and analytically weak solutions as defined e.g. in the book by Liu and Röckner [48, Appendix G]. We also note that a solution is called weak solution in the book by Da Prato and Zabczyk [21].

For $u_{0} \in H$, we may assume that that there exists a unique solution to (2.1) and that $U \in C([0, T] ; H), \mathbb{P}$-a.s., see [44, p. 5] and also [21, Proposition 7.5].

The next step is to study the gradient structure.

\footnotetext{
${ }^{2}$ Or, more generally, with $u_{0} \in L^{2}\left(\Omega, \mathcal{F}_{0}, \mathbb{P} ; L^{2}(\mathcal{B})\right)$.
} 
2.1. Gradients in (nonlocal) Hilbert space. Let us begin with some preliminary considerations. By the spectral theorem, under Assumption 2, $K$ has at most countably many points in its spectrum $\sigma(K)$, all of those being real, nonnegative and zero being their only accumulation point.

Note that upon setting

$$
\bar{g}(x):= \begin{cases}g(x), & x \in \mathcal{B}, \\ 0, & x \in \mathbb{R}^{d} \backslash \mathcal{B},\end{cases}
$$

we have the convolution representation $K g=J * \bar{g}$. Let $\left\{\lambda_{i}\right\}_{i \in \mathbb{N}}$ be the sequence of positive real eigenvalues of $K$ on $H$. In our situation, w.l.o.g. $\lambda_{i} \rightarrow 0$, for $i \rightarrow \infty,\left\{\lambda_{i}\right\} \in \ell^{2}$ and there is a (possibly finite) orthonormal system $\left\{e_{i}\right\}_{i \in \mathbb{N}}$ of eigenfunctions for $K$. By the spectral theorem,

$$
H=\operatorname{ker} K \oplus \overline{\operatorname{span}\left\{e_{i}\right\}}
$$

where the decomposition is orthogonal, cf. [58]. Denote

$$
S:=(\operatorname{ker} K)^{\perp}=\overline{\operatorname{span}\left\{e_{i}\right\}} .
$$

$S$ is a closed Hilbert subspace of $H$. The space $S$ is key to reformulate the problem. The next step is to endow it with a norm. To emphasize this, we set

$$
H_{-1}:=S
$$

and consider the (nonlocal) norm

$$
\|g\|_{-1}:=\|g\|_{H_{-1}}:=\left\|K^{-\frac{1}{2}} g\right\|_{H}, \quad g \in S,
$$

where $K^{-\frac{1}{2}}: S \rightarrow S$ is the operator square root of the Moore-Penrose pseudoinverse $K^{-1}: S \rightarrow S$ of $K: H \rightarrow S$, see [34, Section 2.1.2]. $H_{-1}$ is a separable Hilbert space with nonlocal norm and inner product $(\cdot, \cdot)_{-1}:=\left(K^{-\frac{1}{2}} \cdot, K^{-\frac{1}{2} \cdot} \cdot\right)_{H}$. Note that $K^{-1}$ is nonnegative on $S$ and that $K^{-\frac{1}{2}}$ is the pseudoinverse of $K^{\frac{1}{2}}: H \rightarrow S$. The space $H_{-1}$ will be used below to reformulate the neural field equation. For later use, we also note the relation

$$
\|g\|_{H} \leq\left\|K^{\frac{1}{2}}\right\|_{L(H)}\|g\|_{-1} \quad g \in S,
$$

which just follows from the definitions and where for separable Hilbert spaces $U, V$, we denote by $\|\cdot\|_{L(U, V)}$ the operator norm and we set $\|\cdot\|_{L(U)}:=\|\cdot\|_{L(U, U)}$.

Let us also define $H_{1}:=K(S)$ with norm $\|g\|_{H_{1}}:=\left\|K^{\frac{1}{2}} g\right\|_{H}$. Of course, $\|\cdot\|_{H_{1}}$ is also defined for elements in $H$ or $S$, however, it is zero on ker $K$ and $\left\{h \in S: K^{2} h=0\right\}$.

At this point, we note that our decomposition of the kernel operator $K$ has some similarities to the approximation by so-called Pincherle-Goursat kernels (PG-kernels), see e.g. 69], which serve as finite-dimensional range approximations of $L^{2}$-kernels. Veltz and Faugeras [71 have used the PG-kernel decomposition for the analysis of systems of neural field equations. They utilize the explicit representation of PG-kernels for a reduction of the neural field model considered by them to a finite number of ODEs via an orthogonal decomposition of the eigenspaces (compare also Subsection 2.3 below). One main difference of their approach to ours is that we require our kernels to be nonnegative in order to manage the case of possibly infinitely many distinct eigenvalues while still keeping a Hilbert space setup.

Let $\varphi: \mathbb{R} \rightarrow \mathbb{R}$ be any primitive function/antiderivative of $f$. For example, we may take $\varphi(t):=\int_{0}^{t} f(s) \mathrm{d} s, t \in \mathbb{R}$. Define

and

$$
\Phi(u):=\int_{\mathcal{B}} \varphi(u(x)) \mathrm{d} x, \quad u \in H,
$$

$$
\Psi(u)=\Psi^{\alpha}(u):=\frac{\alpha}{2} \int_{\mathcal{B}}\left|\left(K^{-\frac{1}{2}} u\right)(x)\right|^{2} \mathrm{~d} x=\frac{\alpha}{2}\|u\|_{-1}^{2}, \quad u \in S .
$$


Lemma 2.4. $\Phi$ is well-defined, finite for all $u \in H$ and continuous on $H$. Furthermore, we have that

$$
\mathrm{D} \Phi(u) h=(F(u), h)_{H}, \quad u, h \in H,
$$

where $\mathrm{D} \Phi(u) h$ denotes the Gâteaux-directional derivative of $\Phi$ in $u$ and in direction $h$.

Proof. We start by proving the claimed properties of $\Phi$. By the Lipschitz property of $f$,

$$
|f(r)| \leq|f(0)|+\operatorname{Lip}(f)|r|, \quad r \in \mathbb{R} .
$$

For $r>0$, by the mean-value theorem, there exists $r_{0} \in(0, r)$ with

$$
\varphi(r)-\varphi(0)=r f\left(r_{0}\right) .
$$

Hence

$$
|\varphi(r)| \leq \operatorname{Lip}(f)|r|\left|r_{0}\right|+|f(0)||r|+|\varphi(0)| \leq \operatorname{Lip}(f)|r|^{2}+|f(0)||r|+|\varphi(0)| .
$$

Replacing $r$ by $-r$ yields the same bound for all $r \in \mathbb{R}$. The first claim, in particular, the continuity of $\Phi$ on $H$ follows now by an application of the Nemytskii theorem, see e.g. 64, Theorem II.3.2], that is, $\varphi(\cdot): L^{2}(\mathcal{B}) \rightarrow L^{1}(\mathcal{B})$ gives rise to a continuous nonlinear (Nemytskii) operator.

For the second claim, let $u, h \in H$. Let $t \in \mathbb{R}, t \neq 0$. By the mean-value theorem, for almost every $x \in \mathcal{B}$, there exists $\theta_{x, t} \in(0,1)$ with

$$
\frac{1}{t} \varphi(u(x)+t h(x))-\varphi(u(x))=f\left(u(x)+\theta_{x, t} t h(x)\right) h(x)
$$

and the right-hand side converges to $f(u(x)) h(x)$ as $t \rightarrow 0$ for almost every $x \in \mathcal{B}$. We have that

$$
\frac{1}{t}[\Phi(u+t h)-\Phi(u)]=\frac{1}{t} \int_{\mathcal{B}}[\varphi(u(x)+t h(x))-\varphi(u(x))] \mathrm{d} x .
$$

By the Lipschitz property of $f$ and Lebesgue's dominated convergence theorem, the right hand side converges to $\int_{\mathcal{B}} f(u(x)) h(x) \mathrm{d} x=(F(u), h)_{H}$ as $t \rightarrow 0$.

Lemma 2.4 already indicates, how we might be able deal with the nonlocal term. We define the functional

$$
\Theta(u):=-\Phi \uparrow_{S}(u)+\Psi(u), \quad u \in S,
$$

where $\Phi \uparrow_{S}$ denotes the restriction of $\Phi$ to $S$. $\Theta$ is obviously continuous on $H_{-1}$. Furthermore, we have the following key lemma.

Lemma 2.5. We have that

$$
\mathrm{D} \Phi \uparrow_{S}(u) h=(K F(u), h)_{-1}, \quad u, h \in H_{-1},
$$

where $\mathrm{D} \Phi \uparrow_{S}(u) h$ denotes the Gâteaux-directional derivative of $\Phi \uparrow_{S}$ in $u$ and in direction $h$.

Furthermore, we have that

$$
\mathrm{D} \Theta(u) h=\alpha(u, h)_{-1}-(K F(u), h)_{-1}, \quad u, h \in H_{-1},
$$

where $\mathrm{D} \Theta(u) h$ denotes the Gâteaux-directional derivative of $\Theta$ in $u$ and in direction $h$.

Proof. Clearly, $u, h \in H_{-1} \hookrightarrow H$ and thus by the proof of Lemma 2.4.

$$
\begin{aligned}
\mathrm{D} \Phi(u) h & =(F(u), h)_{H}=\left(K F(u), K^{-1} h\right)_{H} \\
& =\left(K^{-\frac{1}{2}} K F(u), K^{-\frac{1}{2}} h\right)_{H}=(K F(u), h)_{-1}
\end{aligned}
$$

which proves the first claim.

We get from the book by Showalter [64, p. 91], that the Fréchet derivative and thus the Gâteaux-directional derivative of $\Psi$ is given by the following formula $\mathrm{D} \Psi(u) h=\alpha(u, h)_{-1}$. The claim follows now from the preceding discussion, Lemma 2.4 and the above. 
Remark 2.6. In the case that $K$ is nonpositive definite, we can redefine $H_{-1}$ by replacing $K$ by $-K$ in the definition. Now, by changing the sign for $\Theta$ in (2.5), we obtain a gradient by a similar procedure. We can interpret the case of nonnegative definite symmetric kernels as domination by excitation, while the case of nonpositive definite kernels corresponds to domination of the inhibition effects.

2.2. Gradient flow formulation. Having completed the nonlocal functional setting, we now consider the stochastic nonlocal evolution equation

$$
\mathrm{d} V_{t}=-\mathrm{D} \Theta\left(V_{t}\right) \mathrm{d} t+\varepsilon B \mathrm{~d} W_{t}, \quad V_{0}=u_{0} \in H_{-1},
$$

where $\Theta$ is defined as in (2.5) and where $W$ is a cylindrical Wiener process with values in $H$ and $B \in L_{2}(H)$ is nonnegative and symmetric as in the beginning of this section. Due to the change of the ambient space, we give a further definition for a solution to (2.6) as follows.

Definition 2.7. A solution to (2.6) is an $\left\{\mathcal{F}_{t}\right\}$-adapted stochastic process $V \in L^{2}\left(\Omega \times[0, T] ; H_{-1}\right)$ with $V_{0}=u_{0} \in H_{-1}$ and

$$
\left(V_{t}, h\right)_{-1}=\left(u_{0}, h\right)_{-1}-\int_{0}^{t} \mathrm{D} \Theta\left(V_{s}\right) h \mathrm{~d} s+\varepsilon\left(h, B W_{t}\right)_{-1},
$$

for every $h \in H_{-1}$ and every $t \in[0, T]$.

One reason for introducing several notions of solutions is the aim of their formal comparison. In order to justify their applicability, we will prove that the solutions to our gradient-flow formulation indeed coincide with the solution concept for the original formulation of the neural field equations. In particular, in order to prove the invariance of the smaller subspace $H_{-1}$ under the solutions in the original formulation for initial data in $H_{-1}$, we need to assume that the spatial correlations of the noise $W$, which are given by the covariance operator $B$, are sufficiently small relative to the spectrum of $K$ in a summable way, as formally captured in the following hypothesis.

Assumption 4. Assume that

$$
B \in L_{2}\left(H, H_{-1}\right) \quad \text { and } \quad B K^{-1} \in L_{2}\left(H_{-1}, H\right) .
$$

In the case that $K$ and $B$ commute, the two conditions in (2.7) reduce to one, as can be seen as follows.

Example 2.8. Consider the situation that $B \in L_{2}(H)$ is diagonalized with respect to the same orthonormal system as $K$ with eigenvalues

$$
B e_{i}=b_{i} e_{i} \quad i \in \mathbb{N} .
$$

Consider the condition

$$
\left\{b_{i}^{2} \lambda_{i}^{-1}\right\} \in \ell^{1}
$$

Then we claim that (2.8) holds if and only if (2.7) holds.

Indeed, let $B$ have the specific decomposition as assumed. Then $B$ and $K$ (and thus $B$ and $K^{-1}$ ) commute on $S$. Then

$$
\sum_{i=1}^{\infty}\left\|B e_{i}\right\|_{H_{-1}}^{2}=\sum_{i=1}^{\infty}\left(K^{-\frac{1}{2}} B e_{i}, K^{-\frac{1}{2}} B e_{i}\right)_{H}=\sum_{i=1}^{\infty}\left(B^{2} K^{-1} e_{i}, e_{i}\right)_{H}=\sum_{i=1}^{\infty} \frac{b_{i}^{2}}{\lambda_{i}}
$$


and thus the first part of (2.7) holds if and only if (2.8) holds. For the second part, note that $\left\{\sqrt{\lambda_{i}} e_{i}\right\}_{i \in \mathbb{N}}$ is an orthonormal basis for $H_{-1}$, so that

$$
\sum_{i=1}^{\infty}\left\|\sqrt{\lambda_{i}} B K^{-1} e_{i}\right\|_{H}^{2}=\sum_{i=1}^{\infty} \lambda_{i}\left\|\frac{b_{i}}{\lambda_{i}} e_{i}\right\|_{H}^{2}=\sum_{i=1}^{\infty} \frac{b_{i}^{2}}{\lambda_{i}}
$$

Hence (2.8) holds if and only if (2.7) holds.

For instance, $B:=K$ is an example which yields relation (2.8), as $K$ is of trace class in $H$ by Mercer's theorem, see also [15, p. 91, equation (6.55)].

We can compare the solutions to (2.1) and (2.6) in the following way. The mentioned invariance result is postponed to the next subsection.

Proposition 2.9. Suppose that $u_{0} \in H_{-1}$. Under Assumptions 1-4, a solution to (2.1) in the sense of Definition 2.1 is a solution to (2.6) in the sense of Definition 2.7.

Proof. Let $U$ be a solution to (2.1). By Theorem 2.12 below, we see that $u_{0} \in H_{-1}$ and the assumed relation (2.7) imply that

$$
U \in L^{2}\left(\Omega ; L^{\infty}\left([0, T] ; H_{-1}\right)\right) .
$$

For any $h \in H_{-1}$ there exists $v \in H$ with $K v=h$. We see that

$$
\left(U_{t}, v\right)_{H}=\left(u_{0}, v\right)_{H}+\int_{0}^{t}\left(-\alpha U_{s}+K F\left(U_{s}\right), v\right)_{H} \mathrm{~d} s+\varepsilon\left(v, B W_{t}\right)_{H},
$$

is equivalent to

$$
\begin{aligned}
& \left(U_{t}, K^{-1} h\right)_{H} \\
= & \left(u_{0}, K^{-1} h\right)_{H}+\int_{0}^{t}\left(-\alpha U_{s}+K F\left(U_{s}\right), K^{-1} h\right)_{H} \mathrm{~d} s+\varepsilon\left(K^{-1} h, B W_{t}\right)_{H} .
\end{aligned}
$$

Thus, just using the definitions of the nonlocal norms, we get

$$
\left(U_{t}, h\right)_{-1}=\left(u_{0}, h\right)_{-1}+\int_{0}^{t}\left(-\alpha U_{s}+K F\left(U_{s}\right), h\right)_{-1} \mathrm{~d} s+\varepsilon\left(h, B W_{t}\right)_{-1} .
$$

Finally, the representation of $\mathrm{D} \Theta(\cdot)$, proved above, yields

$$
\left(U_{t}, h\right)_{-1}=\left(u_{0}, h\right)_{-1}-\int_{0}^{t} \mathrm{D} \Theta\left(U_{s}\right) h \mathrm{~d} s+\varepsilon\left(h, B W_{t}\right)_{-1} .
$$

Corollary 2.10. Suppose that $u_{0} \in H_{-1}$. Assume that Assumptions 1-4 hold. Then a solution to (2.6) in the sense of Definition 2.7 is a solution to (2.1) in the sense of Definition 2.1.

Proof. This is proved by reading the chain of equations in the proof of Proposition 2.9 backwards.

Although we now know that solutions are indeed equivalent, we have to show that they stay in the nonlocal space $H_{-1}$ if they start in $H_{-1}$ as our gradient flow formulation is only valid for this space. 
2.3. Galerkin approximation and invariance of $H_{-1}$ under the flow. The idea to show invariance is to use a finite-system approximation idea in combination with Itô's formula. Consider $u_{0} \in H$ and let $u^{i, N}, N \in \mathbb{N}, 1 \leq i \leq N$ be a solution to the $N$-dimensional Galerkin system of stochastic ordinary differential equations

$$
\mathrm{d} u_{t}^{i, N}=\left[-\alpha u_{t}^{i, N}+(K F)^{i, N}\left(u_{t}^{1, N}, \ldots, u_{t}^{N, N}\right)\right] \mathrm{d} t+\varepsilon\left(B e_{i}, \mathrm{~d} W_{t}\right)_{H}, \quad u_{0}^{i, N}=u_{0},
$$

for each $1 \leq i \leq N$. Here, $(K F)^{i, N}$ are given by

$$
\begin{aligned}
& (K F)^{i, N}\left(u_{t}^{1, N}, \ldots, u_{t}^{N, N}\right) \\
:= & \int_{\mathcal{B}} f\left(\sum_{j=1}^{N} u^{j, N}(x) e_{j}(x)\right)\left(\int_{\mathcal{B}} J(x-y) e_{i}(y) \mathrm{d} y\right) \mathrm{d} x,
\end{aligned}
$$

compare with [44, Section 8]. Note that we can replace each of the noise terms $\varepsilon\left(B e_{i}, \mathrm{~d} W_{t}\right)_{H}$ by multiples of independent real-valued Brownian motions $\varepsilon b_{i} \mathrm{~d} \beta_{t}^{i}$ if the cylindrical Wiener process $W$ is modeled with respect to the orthonormal system $\left\{e_{i}\right\}$ and if $B$ and $K$ are codiagonal, see Example 2.8. As we do not want to assume this for the sake of generality, we shall accept the constraint that the modes of the noise may be probabilistically coupled (solutions to the Galerkin system exist nevertheless). Let $U$ be a solution to (2.1). The $N$ th Galerkin approximation $U^{N}$ to $U$ is defined by

$$
U_{t}^{N}:=\sum_{i=1}^{N} u_{t}^{i, N} e_{i}
$$

The following theorem can be found in [44, Theorem 8.1].

Theorem 2.11. For every $T>0$, it holds that

$$
\lim _{N \rightarrow \infty}\left[\sup _{t \in[0, T]}\left\|U_{t}-U_{t}^{N}\right\|_{H}\right]=0 \quad \mathbb{P} \text {-a.s. }
$$

Having finished our preparations we can now show that if we choose the initial datum $u_{0} \in$ $H_{-1}$, we remain in the subspace $H_{-1}$. This is a consequence of the following invariance result.

Theorem 2.12. Let $u_{0} \in H_{-1}$. Assume that Assumptions 1-4 hold. Then $U \in L^{2}(\Omega \times$ $\left.[0, T] ; H_{-1}\right)$ and there exists a constant $C>0$ such that

$$
\mathbb{E}\left[\sup _{t \in[0, T]}\left\|U_{t}\right\|_{-1}^{2}\right] \leq C\left\|u_{0}\right\|_{-1}^{2}+\varepsilon^{2} C
$$

where $\varepsilon \geq 0$ is as in (2.1).

Proof. Note that since $u_{0} \in H_{-1}$, we may assume that $U^{N} \in C\left([0, T] ; H_{-1}\right) \mathbb{P}$-a.s., see the discussion by Kuehn and Riedler [44, Sections 3 and 8]. Also, in this case,

$$
(K F)^{i, N}\left(u_{t}^{1, N}, \ldots, u_{t}^{N, N}\right)=\lambda_{i} \int_{\mathcal{B}} f\left(\sum_{j=1}^{N} u^{j, N}(x) e_{j}(x)\right) e_{i}(x) \mathrm{d} x .
$$

We also have to consider the truncated action of the kernel defined by

$$
K^{N} v:=\sum_{i=1}^{N} \lambda_{i}\left(e_{i}, v\right)_{H} e_{i} .
$$


Applying Itô's formula (see [21, Theorem 4.32]) for the functional $v \mapsto\|v\|_{-1}^{2}$ yields

$$
\begin{aligned}
\left\|U_{t}^{N}\right\|_{-1}^{2}= & \left\|u_{0}\right\|_{-1}^{2}+2 \int_{0}^{t}\left(-\alpha U_{s}^{N}+K^{N} F\left(U_{s}^{N}\right), U_{s}^{N}\right)_{-1} \mathrm{~d} s \\
& +2 \varepsilon \sum_{i=1}^{N} \int_{0}^{t}\left(U_{s}^{N}, B^{i} e_{i} \mathrm{~d} \beta_{s}^{i}\right)_{-1}+t \varepsilon^{2} \sum_{i=1}^{N} \sum_{k=1}^{N}\left(B e_{i}, e_{k}\right)_{H}^{2} \lambda_{i}^{-1} .
\end{aligned}
$$

Note that $K^{N}$ is a nonnegative definite operator. We see that $\mathrm{d} t \otimes \mathbb{P}$-a.e., by the spectral decomposition of $U^{N}$ and $K^{-1}$ and orthogonality,

$$
\begin{aligned}
& \left(-\alpha U^{N}+K^{N} F\left(U^{N}\right), U^{N}\right)_{-1} \\
= & -\alpha\left\|U^{N}\right\|_{-1}^{2}+\left(\sum_{j=1}^{N} \lambda_{j}\left(e_{j}, F\left(U^{N}\right)\right)_{H} e_{j}, K^{-1} U^{N}\right)_{H} \\
= & -\alpha\left\|U^{N}\right\|_{-1}^{2}+\left(\sum_{j=1}^{N} \lambda_{j}\left(e_{j}, F\left(U^{N}\right)\right)_{H} e_{j}, \sum_{k=1}^{N} \lambda_{k}^{-1}\left(U^{N}, e_{k}\right)_{H} e_{k}\right)_{H} \\
= & -\alpha\left\|U^{N}\right\|_{-1}^{2}+\left(F\left(U^{N}\right), U^{N}\right)_{H} \\
\leq & -\alpha\left\|U^{N}\right\|_{-1}^{2}+[|f(0)|+\operatorname{Lip}(f)]\|K\|_{L(H)}\left\|U^{N}\right\|_{-1}^{2},
\end{aligned}
$$

where we have used (2.4) and (2.3). To bound $U_{t}^{N}$ uniformly in $t \in[0, T]$, we are first going to use the Burkholder-Davis-Gundy inequality, the Young inequality, and Gronwall's lemma to show that there exist constants $C:=[|f(0)|+\operatorname{Lip}(f)]\|K\|_{L(H)}>0, \delta \in(0,1), \delta<\frac{1}{2 T} \mathrm{e}^{2(\alpha-C) T}$ and $\kappa(\delta)>0$, such that the following inequality holds, i.e., we estimate (2.12) as follows

$$
\begin{aligned}
& \mathbb{E}\left[\sup _{t \in[0, T]}\left\|U_{t}^{N}\right\|_{-1}^{2}\right] \\
\leq & \mathrm{e}^{2(C-\alpha) T}\left\|u_{0}\right\|_{-1}^{2}+\mathrm{e}^{2(C-\alpha) T} \delta \mathbb{E} \int_{0}^{T}\left\|U_{s}^{N}\right\|_{-1}^{2} \mathrm{~d} s \\
& +\mathrm{e}^{2(C-\alpha) T} T \varepsilon^{2} \kappa(\delta) \sum_{i=1}^{N}\left\|B e_{i}\right\|_{H_{-1}}^{2} \\
& +\mathrm{e}^{2(C-\alpha) T} T \varepsilon^{2} \sum_{i=1}^{N} \sum_{k=1}^{N}\left(B e_{i}, e_{k}\right)_{H}^{2} \lambda_{i}^{-1} \\
\leq & \mathrm{e}^{2(C-\alpha) T}\left\|u_{0}\right\|_{-1}^{2}+\frac{1}{2} \mathbb{E}\left[\sup _{s \in[0, T]}\left\|U_{s}^{N}\right\|_{-1}^{2}\right] \\
& \left.+\mathrm{e}^{2(C-\alpha) T} T \varepsilon^{2} \kappa(\delta)\|B\|_{L_{2}\left(H, H_{-1}\right)}^{2}\right) \\
& +\mathrm{e}^{2(C-\alpha) T} T \varepsilon^{2} \sum_{i=1}^{N}\left(B e_{i}, B e_{i}\right)_{H} \lambda_{i}^{-1}
\end{aligned}
$$

where we have used Bessel's inequality in the last step. Clearly, noting that $\left\{\sqrt{\lambda_{i}} e_{i}\right\}_{i \in \mathbb{N}}$ is an orthonormal basis for $H_{-1}$, we get for every $N \in \mathbb{N}$,

$$
\sum_{i=1}^{N}\left(B e_{i}, B e_{i}\right)_{H} \lambda_{i}^{-1}=\sum_{i=1}^{N}\left\|\sqrt{\lambda_{i}} B K^{-1} e_{i}\right\|_{H}^{2} \leq\left\|B K^{-1}\right\|_{L_{2}\left(H_{-1}, H\right)}^{2}
$$


and hence by (2.7), we get that there exists another constant $C>0$ with

$$
\mathbb{E}\left[\sup _{t \in[0, T]}\left\|U_{t}^{N}\right\|_{-1}^{2}\right] \leq C\left\|u_{0}\right\|_{-1}^{2}+\varepsilon^{2} C
$$

for each $N \in \mathbb{N}$ and thus

$$
\mathbb{E}\left[\sup _{N \in \mathbb{N}} \sup _{t \in[0, T]}\left\|U_{t}^{N}\right\|_{-1}^{2}\right] \leq C\left\|u_{0}\right\|_{-1}^{2}+\varepsilon^{2} C .
$$

Hence $\left\{U^{N}\right\}$ has a subsequence $\left\{U^{N_{k}}\right\}$ that converges in the weak* sense in the Banach space $L^{2}\left(\Omega ; L^{\infty}\left([0, T] ; H_{-1}\right)\right)$ to some element $\tilde{U} \in L^{2}\left(\Omega ; L^{\infty}\left([0, T] ; H_{-1}\right)\right)$. Since $H_{-1}$ is continuously embedded into $H$, we see by Theorem 2.11 that $\tilde{U}=U$ holds $\mathrm{d} t \otimes \mathbb{P}$-a.e.. We conclude the proof by noting that the $L^{2}\left(\Omega ; L^{\infty}\left([0, T] ; H_{-1}\right)\right)$-norm is lower semi-continuous with respect to weak*-convergence. Hence, we can pass on to the limit $N \rightarrow \infty$ in (2.14) and get that

$$
\mathbb{E}\left[\sup _{t \in[0, T]}\left\|U_{t}\right\|_{-1}^{2}\right] \leq C\left\|u_{0}\right\|_{-1}^{2}+\varepsilon^{2} C
$$

which indeed just means that the solution stays in $H_{-1}$.

2.4. Pathwise regularity of the flow. In addition to the construction of the flow, it is often helpful, sometimes even imperative, to have finer control over its regularity. Let $V \in$ $L^{2}\left(\Omega \times[0, T] ; H_{-1}\right)$ be a solution to (2.6) for the initial datum $u_{0} \in H_{-1}$. Consider the DossSussmann transformation

$$
Y:=V-\varepsilon B W
$$

which is an established tool for certain classes of stochastic evolution equations [18]. Then $Y$ satisfies the random evolution equation

$$
\mathrm{d} Y_{t}=-\mathrm{D} \Theta\left(Y_{t}+\varepsilon B W_{t}\right) \mathrm{d} t, \quad Y_{0}=u_{0},
$$

where $\Theta$ is defined as in (2.5). If $Y$ is a pathwise solution for (2.15), we may transform back by setting $V(\omega)=Y(\omega)+\varepsilon B W(\omega)$ for each $\omega \in \Omega$. Note that we may have to drop the assumption that the filtered probability space is normal in order to obtain a collection of paths which fits our purposes. We shall use the formulation of (2.15) in order to prove additional regularity for the gradient flow $V$.

Proposition 2.13. Suppose that for fixed $\omega \in \Omega, t \mapsto B W_{t}(\omega)$ is càdlàg in $H_{-1}$ and that

$$
B W(\omega) \in L^{2}\left([0, T] ; H_{-1}\right)
$$

Let $V$ be a solution to (2.6) for the initial datum $u_{0} \in H_{-1}$. Then the map $t \mapsto V_{t}$ is weakly continuous in $H_{-1}$ and strongly right-continuous in $H_{-1}$.

Proof. The strategy of the argument can, e.g., be found in another setting in the work by Gess and Tölle [32, Proof of Theorem 2.6]. Since we work with a random evolution equation pathwise, we may apply the chain rule [64, Section III.4] for the functional $v \mapsto\|v\|_{-1}^{2}$ such that for any

\footnotetext{
${ }^{3}$ That is, right-continuous with left limits.
} 
$\kappa \geq 0$,

$$
\begin{aligned}
& \left\|Y_{t}\right\|_{-1}^{2} \mathrm{e}^{-\kappa t} \\
& =\left\|u_{0}\right\|_{-1}^{2}-2 \int_{0}^{t} \mathrm{e}^{-\kappa s}\left(\mathrm{D} \Theta\left(Y_{s}+\varepsilon B W_{s}\right), Y_{s}\right)_{-1} \mathrm{~d} s-\kappa \int_{0}^{t} \mathrm{e}^{-\kappa s}\left\|Y_{s}\right\|_{-1}^{2} \mathrm{~d} s \\
& \leq\left\|u_{0}\right\|_{-1}^{2}-2 \alpha \int_{0}^{t} \mathrm{e}^{-\kappa s}\left\|Y_{s}+\varepsilon B W_{s}\right\|_{-1}^{2} \mathrm{~d} s \\
& +2 \alpha \varepsilon^{2} \int_{0}^{t} \mathrm{e}^{-\kappa s}\left(Y_{s}+B W_{s}, B W_{s}\right)_{-1} \mathrm{~d} s \\
& \quad+2[|f(0)|+\operatorname{Lip}(f)]\|K\|_{L(H)} \int_{0}^{t} \mathrm{e}^{-\kappa s}\left\|Y_{s}+\varepsilon B W_{s}\right\|_{-1}\left\|Y_{s}\right\|_{-1} \mathrm{~d} s \\
& \quad-\kappa \int_{0}^{t} \mathrm{e}^{-\kappa s}\left\|Y_{s}\right\|_{-1}^{2} \mathrm{~d} s \\
& \leq\left\|u_{0}\right\|_{-1}^{2}+\frac{\alpha \varepsilon^{2}}{2} \int_{0}^{t} \mathrm{e}^{-\kappa s}\left\|B W_{s}\right\|_{-1}^{2} \mathrm{~d} s \\
& +C \int_{0}^{t} \mathrm{e}^{-\kappa s}\left\|Y_{s}\right\|_{-1}^{2} \mathrm{~d} s+C \varepsilon^{2} \int_{0}^{t} \mathrm{e}^{-\kappa s}\left\|B W_{s}\right\|_{-1}^{2} \mathrm{~d} s-\kappa \int_{0}^{t} \mathrm{e}^{-\kappa s}\left\|Y_{s}\right\|_{-1}^{2} \mathrm{~d} s ;
\end{aligned}
$$

compare with (2.13). Choosing $\kappa=C$ yields

$$
\left\|Y_{t}\right\|_{-1}^{2} \mathrm{e}^{-C t} \leq\left\|u_{0}\right\|_{-1}^{2}+\varepsilon^{2}\left(C+\frac{\alpha}{2}\right) \int_{0}^{t} \mathrm{e}^{-C s}\left\|B W_{s}\right\|_{-1}^{2} \mathrm{~d} s,
$$

which is finite by (2.16). As, in particular,

$$
\sup _{t \in[0, T]}\left\|Y_{t}\right\|_{-1}^{2}<\infty
$$

and since $t \mapsto Y_{t}=V_{t}-B W_{t}$ is continuous in $H$, we get by the continuous embedding $H_{-1} \hookrightarrow H$ that $t \mapsto Y_{t}$ is continuous with respect to the weak topology in $H_{-1}$. Note that by the same calculation as in (2.17), we get that

$$
\left\|Y_{t}\right\|_{-1}^{2} \mathrm{e}^{-C t} \leq\left\|Y_{s}\right\|_{-1}^{2} \mathrm{e}^{-C s}+\varepsilon^{2}\left(C+\frac{\alpha}{2}\right) \int_{s}^{t} \mathrm{e}^{-C r}\left\|B W_{r}\right\|_{-1}^{2} \mathrm{~d} r,
$$

for $T \geq t>s \geq 0$. Let $t_{n} \in[0, T]$ with $t_{n} \searrow t$. We find that

$$
\left\|Y_{t_{n}}\right\|_{-1}^{2} \mathrm{e}^{-C t_{n}} \leq\left\|Y_{t}\right\|_{-1}^{2} \mathrm{e}^{-C t}+\varepsilon^{2}\left(C+\frac{\alpha}{2}\right) \int_{t}^{t_{n}} \mathrm{e}^{-C s}\left\|B W_{s}\right\|_{-1}^{2} \mathrm{~d} s
$$

and thus

$$
\limsup _{n \rightarrow \infty}\left\|Y_{t_{n}}\right\|_{-1}^{2} \leq\left\|Y_{t}\right\|_{-1}^{2} .
$$

By weak continuity in $H_{-1}$, we obtain that $Y_{t_{n}} \rightarrow Y_{t}$ in $H_{-1}$. Since $t \mapsto B W_{t}$ is right-continuous in $H_{-1}$, we get that $V=Y+\varepsilon B W$ is right-continuous in $H_{-1}$.

\section{ERGODICITY AND UNIQUE INVARIANT MEASURES}

We are now going to exploit the gradient structure for the neural field equation (1.1). In particular, we are going to apply classical results, which allow for a direct computation of the unique invariant measure of the ergodic semigroup of the (stochastic) flow, see e.g. 50, 51]. To apply these results in our setting, let $V^{x}:[0, T] \times \Omega \rightarrow H_{-1}$ be the unique solution to (2.6) 
with initial datum $V_{0}^{x}=x \in H_{-1}$. Assume $\varepsilon>0$ here, which rules out the situation of the deterministic PDE. Define the semigroup

$$
P_{t}^{\varepsilon} G(x):=\mathbb{E}\left[G\left(V_{t}^{x}\right)\right] \quad G \in \mathcal{B}_{b}\left(H_{-1}\right), x \in H_{-1}, t \geq 0,
$$

where, for a topological space $X, \mathcal{B}_{b}(X)$ denotes the space of bounded Borel measurable maps from $X$ to $\mathbb{R}$.

Definition 3.1. We say that $\left\{P_{t}^{\varepsilon}\right\}_{t \geq 0}$ is symmetric with respect to a probability measure $\nu$ on $\left(H_{-1}, \mathcal{B}\left(H_{-1}\right)\right)$ i用

$$
\int_{H_{-1}} G_{1}(x) P_{t}^{\varepsilon} G_{2}(x) \nu(\mathrm{d} x)=\int_{H_{-1}} G_{2}(x) P_{t}^{\varepsilon} G_{1}(x) \nu(\mathrm{d} x) \quad \forall G_{1}, G_{2} \in \mathcal{B}_{b}\left(H_{-1}\right)
$$

for every $t \geq 0$.

Remark 3.2. If $\left\{P_{t}^{\varepsilon}\right\}_{t \geq 0}$ is symmetric with respect to some $\nu$, then $\nu$ is an invariant measure for $\left\{P_{t}^{\varepsilon}\right\}_{t \geq 0}$, that is,

$$
\left(P_{t}^{\varepsilon}\right)^{*} \nu=\nu \quad \forall t \geq 0
$$

where

$$
\left(P_{t}^{\varepsilon}\right)^{*} \nu(E):=\int_{H_{-1}} P_{t}^{\varepsilon} 1_{E} \nu(\mathrm{d} x), \quad E \in \mathcal{B}\left(H_{-1}\right)
$$

is the dual semigroup, see [20] for details regarding these standard semigroup constructions.

Note that we can write equation (2.6) also in the form

$$
\mathrm{d} X_{t}=\left(A X_{t}+\mathrm{D} \Phi\left(X_{t}\right)\right) \mathrm{d} t+\varepsilon B \mathrm{~d} W_{t}, \quad X_{0}=x \in H_{-1},
$$

where $A$ corresponds to the linear decay term.

Clearly, $A$ generates a $C_{0}$-contraction semigroup $\left\{S_{t}\right\}_{t \geq 0}$ on $H_{-1}=\left(S,\|\cdot\|_{-1}\right)$ which is extendable to a $C_{0}$-contraction semigroup $\left\{S_{t}^{0}\right\}_{t \geq 0}$ on the subset $\left(S,\|\cdot\|_{H}\right)$ of $H$ by the spectral theorem. The infinitesimal generator of $\left\{S_{t}^{0}\right\}_{t \geq 0}$ is denoted by $A^{0}$. By the definition of the space $H_{-1}, A^{0}$ is a realization of $-\alpha K^{-1}$.

Also note that by our assumptions above, $A^{0}$ is self-adjoint and on $\left(S,\|\cdot\|_{H}\right)$ and nonpositive definite. Set

$$
\Gamma_{\varepsilon}:=\frac{\varepsilon^{2}}{2}\left(-A^{0}\right)^{-1}=\frac{2 \varepsilon^{2}}{\alpha} K,
$$

which is a trace class (i.e., a nuclear) operator. Denote by $\gamma_{\varepsilon} \sim N\left(0, \Gamma_{\varepsilon}\right)$ the centered Gaussian measure with covariance operator $\Gamma_{\varepsilon}$, which is concentrated on $S$, see e.g. [11].

Theorem 3.3. Assume that Assumptions 1-4 hold and that $B=K$. Then we get that the semigroup $\left\{P_{t}^{\varepsilon}\right\}_{t \geq 0}$ is strongly Markovian 5 and symmetric with respect to the measure

$$
\mu^{\varepsilon}(\mathrm{d} z):=Z_{\varepsilon}^{-1} \exp \left(2 \varepsilon^{-2} \Phi(z)\right) \gamma_{\varepsilon}(\mathrm{d} z),
$$

where $Z_{\varepsilon}:=\int_{S} \exp \left(2 \varepsilon^{-2} \Phi(z)\right) \gamma_{\varepsilon}(\mathrm{d} z)$. In particular, $\mu^{\varepsilon}$ is an invariant measure for $\left\{P_{t}^{\varepsilon}\right\}_{t \geq 0}$.

Proof. We have the correct structure for our equation enabling us to apply [75, Theorem 2]. Note that "Assumption (H3)" in [75] follows from [21, Theorem 7.14], combined with [21, Theorem 9.21]. We refer also to [19] for an historical account. Compare also with [55, Theorem 5].

\footnotetext{
${ }^{4}$ For a topological space $X, \mathcal{B}(X)$ denotes the Borel $\sigma$-algebra.

${ }^{5}$ See [21, Chapter 9] for the definition of this notion.
} 
Of course, we recognize the invariant measure (3.1) as the usual Gibbs measure well-known from statistical physics, yet here it simply "lives" on a nonlocal space. However, its biophysical relevance is the same as the classical one as it can be interpreted as the stationary probability of a state. In fact, even more can be said about $\mu^{\varepsilon}$. Under the assumptions above, the measure $\mu^{\varepsilon}$ is in fact unique and the semigroup $\left\{P_{t}^{\varepsilon}\right\}_{t \geq 0}$ is ergodic. Recall that $\delta_{x}(B):=1_{B}(x), B \in \mathcal{B}\left(H_{-1}\right)$ denotes the Dirac measure and $\|\cdot\|_{\mathrm{TV}}$ denotes the total variation of a measure.

Theorem 3.4. Assume that Assumptions 1-4 hold and that $B=K$. Then the measure $\mu^{\varepsilon}$, as defined in (3.1), is unique and the semigroup $\left\{P_{t}^{\varepsilon}\right\}_{t \geq 0}$ is ergodic and strong Feller in the following restricted sense:

$$
\left\|\left(P_{t}^{\varepsilon}\right)^{*} \delta_{x_{n}}-\left(P_{t}^{\varepsilon}\right)^{*} \delta_{x_{0}}\right\|_{\mathrm{TV}} \rightarrow 0
$$

as $n \rightarrow \infty$ for any sequence of points $x_{n} \in H_{-1}, n \in \mathbb{N}, x_{0} \in H_{-1}$ with $\left\|x_{n}-x_{0}\right\|_{-1} \rightarrow 0$ as $n \rightarrow \infty$ and for any $t>0$.

Proof. See [52, Theorem 2.1], where "Assumption (H4)" follows in [52] follows from [21, Theorem 7.14], combined with [21, Theorem 9.21].

Uniqueness of the invariant measure (3.1) then follows from its so-called asymptotic strong Feller property, see [35, 36] for this notion.

Corollary 3.5. Assume that Assumptions $1-4$ hold and that $B=K$. Then $\left\{P_{t}^{\varepsilon}\right\}_{t \geq 0}$ is asymptotic strong Feller.

Proof. See [35, Remark 3.9].

Remark 3.6. The requirement that $B=K$ is not necessary for the existence of invariant measures. We have assumed this condition in order to get an explicit and simple representation for the invariant measure. In a finite dimensional context, this observation goes back to Kolmogorov [41]. In fact, in order to obtain a symmetrizing Gibbs-type representation for the invariant measure as in (3.1), it is necessary and sufficient to require $B=K, c f$. the exposition by Mück [55]. By an application Krylov-Bogoliubov's method, we note that under Assumptions 1-4, there always exists an invariant measure, see e.g. [20, 277].

Under additional monotonicity and weak dissipativity assumptions on $f$ one might even obtain decay estimates on invariant measures as e.g. in the works by Barbu and Da Prato [5], Liu and Tölle [49]. However, these steps are beyond the basic framework we develop here.

\section{Examples of NONNEGative Definite KeRnels}

To illustrate the setting, we also have to provide several concrete examples. The key restriction that is required for the gradient structure setup is the nonnegative definiteness assumption for the kernel. Let us recall the following useful generating function characterization of positive definite kernels determined by a function $J$ as in Assumption 2 ,

Theorem 4.1. A kernel of the form $(x, y) \mapsto J(x-y), x, y \in \mathbb{R}^{d}$, for some function $J: \mathbb{R}^{d} \rightarrow \mathbb{R}$, is nonnegative definite in the sense of (2.2) if and only if there exists a finite nonnegative Borel measure $\hat{\sigma}$ on $\left(\mathbb{R}^{d}, \mathcal{B}\left(\mathbb{R}^{d}\right)\right)$ for which

$$
J(x)=\int_{\mathbb{R}^{d}} \mathrm{e}^{\mathrm{i}\langle y, x\rangle} \hat{\sigma}(\mathrm{d} y), \quad x \in \mathbb{R}^{d} .
$$

Proof. See [30, Theorem 7.5] and also [62, 66]. 
This result can be applied under the caveat that it essentially holds for $\mathbb{R}^{d}$, but we need to truncate and rescale accordingly (while restricting to radially symmetric support) in order to adapt it to use in our context.

We shall give a couple of classical examples.

Example 4.2. The following examples for $J$ satisfy relation [4.1] for some $\hat{\sigma}$, see e.g. [69].

(i) (centered Gauss distribution) $J(x)=\mathrm{e}^{-\frac{1}{2}\langle x, M x\rangle}$, where $M \in \mathbb{R}^{d \times d}$ is a symmetric and positive semi-definite matrix.

(ii) (centered Cauchy distribution) $J(x)=\mathrm{e}^{-\sqrt{\langle x, M x\rangle}}$, where $M \in \mathbb{R}^{d \times d}$ is a symmetric and positive semi-definite matrix.

(iii) (centered Laplace distribution) $J(x)=\left(1+\frac{1}{2}\langle x, M x\rangle\right)^{-1}$, where $M \in \mathbb{R}^{d \times d}$ is a symmetric and positive semi-definite matrix.

(iv) (uniform distribution on $\left.[-1,1]^{d}\right) J(x)=J\left(x_{1}, \ldots, x_{d}\right)=\prod_{j=1}^{d} \frac{\sin \left(x_{j}\right)}{x_{j}}$, where the factors of the product are (by definition) equal to 1 if $x_{j}=0$.

(v) (symmetric sums of Dirac distributions) $J(x)=\sum_{i=1}^{\infty} a_{i} \cos \left(\left\langle m_{i}, x\right\rangle\right)$, where $a_{i} \geq 0$ with $\sum_{i=1}^{\infty} a_{i}=1$ and $m_{i} \in \mathbb{R}^{d}, m_{i} \neq \pm m_{j}$ for $i \neq j$.

In fact, by [62, Theorem 1.3.13], relation (4.1) can be verified for some $J$ with the required properties for any probability measure $\hat{\sigma}$ on $\left(\mathbb{R}^{d}, \mathcal{B}\left(\mathbb{R}^{d}\right)\right)$ which is symmetric, that is, $\hat{\sigma}(B)=$ $\hat{\sigma}(-B)$ for all $B \in \mathcal{B}\left(\mathbb{R}^{d}\right)$. These are precisely the distributions of random vectors $X$ for which it holds that $X$ and $-X$ have the same distribution. In this case, we have that

$$
J(x)=\int_{\mathbb{R}^{d}} \cos (\langle y, x\rangle) \hat{\sigma}(\mathrm{d} y), \quad x \in \mathbb{R}^{d},
$$

see [62, Theorem 1.3.13] again. Hence, we can always use the Fourier relation (4.1) to (explicitly or numerically) check, whether our gradient-structure formulation applies. The characterization extends of course to positive multiples of $\hat{\sigma}$.

For instance, by Example 4.2 (ii), we see that the exponential weight used e.g. by Bressloff 14, Equation (9.49)] satisfies our assumptions. Also, by Example 4.2 (v), we see that a finite sum of cosine functions, as considered e.g. by Veltz and Faugeras [71, Section 5] for the so-called periodic ring model introduced by Shriki et al. [65], satisfies our requirements. The cosine weight with period matching the domain size can also be found in [25], we note, however, that the (non-Lipschitz) Heaviside activation function used therein is out of the scope of our paper.

Remark 4.3. (i) We also point out in this context that there seems to be potential for confusion in the literature regarding nonnegative definiteness of certain classes of kernels commonly used in neural fields. For example, consider the (one-dimensional) Mexican hat kernel

$$
J(x)=\left(1-x^{2}\right) \exp \left(-\frac{x^{2}}{2}\right)
$$

which can easily be normalized by a positive pre-factor, scaled in the spatial variable and/or extended into higher dimensions. In a work related to neuroscience one finds the statement that "the Mexican hat kernel differs from the other kernels, since it is not positive definite" [4]. While in certain works on machine learning one finds that "Mexican hat kernel(s) are Mercer kernel(s)" [74], which just implicitly means that the Mexican hat kernel is nonnegative definite (or positive semi-definite). Indeed, the second statement seems correct in view of 4.1) as shown by the simple calculation

$$
J(x)=\left(1-x^{2}\right) \exp \left(-\frac{x^{2}}{2}\right)=\frac{1}{\sqrt{2 \pi}} \int_{\mathbb{R}} \mathrm{e}^{\mathrm{i} \xi x} \exp \left(-\frac{\xi^{2}}{2}\right) \xi^{2} \mathrm{~d} \xi
$$


so that Fourier inversion is possible, i.e., there even exists a measure $\hat{\sigma}$ with an explicit nonnegative density.

(ii) Let us also discuss another family of kernels, prominent in the literature of neural fields, with a similar shape than that of (4.2), which are therefore also known as Mexican hat kernels. Let $0<A<1, s>1$ and consider

We get that

$$
J(x)=\exp \left(-\frac{x^{2}}{2}\right)-A \exp \left(-\frac{x^{2}}{s^{2}}\right) .
$$

$$
J(x)=\frac{1}{\sqrt{2 \pi}} \int_{\mathbb{R}} \mathrm{e}^{\mathrm{i} \xi x}\left[\exp \left(-\frac{\xi^{2}}{2}\right)-\frac{A s}{\sqrt{2}} \exp \left(-\frac{s^{2} \xi^{2}}{4}\right)\right] \mathrm{d} \xi .
$$

In order that $J$ is given by some nonnegative Borel measure as in (4.1), we require that its density, as given inside the square brackets in (4.5), is nonnegative, which, by an elementary computation, is true if and only if $\sqrt{2} \leq s \leq \frac{\sqrt{2}}{A}$.

(iii) In fact, Coombes et al. [17, Equation (1.29)] give yet another example of a kernel representing short range excitation and long-range inhibition (that is, a Mexican hat kernel). Namely, for $0<\Gamma<1, \gamma_{1}>\gamma_{2}>0$,

$$
J(x)=\exp \left(-\gamma_{1}|x|\right)-\Gamma \exp \left(-\gamma_{2}|x|\right) \text {. }
$$

We have that

$$
J(x)=\frac{1}{\sqrt{2 \pi}} \int_{\mathbb{R}} \mathrm{e}^{\mathrm{i} \xi x}\left[2\left(\frac{\gamma_{1}}{\gamma_{1}^{2}+\xi^{2}}-\Gamma \frac{\gamma_{2}}{\gamma_{2}^{2}+\xi^{2}}\right)\right] \mathrm{d} \xi,
$$

see also [17, Equation (1.30)]. It can be seen easily, that the density inside the square brackets in (4.6) is nonnegative if and only if $\Gamma \leq \frac{\gamma_{2}}{\gamma_{1}}$.

(iv) Laing [45, Equation (5.7)] suggests the following kernels (among others), for $b>0$,

$$
J(x)=\mathrm{e}^{-b|x|}(b \sin (|x|)+\cos (x))
$$

and the wizard hat (see [45, p. 157])

$$
J(x)=\frac{1}{4}(1-|x|) \mathrm{e}^{-|x|} .
$$

As discussed already by Laing [45], both examples have a nonnegative Fourier transform and can hence be considered within the scope of our this work.

\section{Summary \& Discussion}

In this work we have proved that the stochastic Amari neural field model has a gradient flow structure for certain types of neural connectivity kernels. We have used this structure to show well-posedness of the model in a nonlocal Hilbert space build from the connectivity kernel. Furthermore, we have shown the existence of unique Gibbs-type invariant measures for the associated Feller semigroup. These results provide a strong indication that neural field models can be analyzed using gradient flow techniques if one builds the actual gradient flow space using the neural connectivity pattern via a kernel. Yet, for certain kernels, we conjecture that there is actually no gradient flow structure, even in an adapted nonlocal Hilbert space. In fact, it seems plausible to expect this from a neuroscience perspective as we also have to allow for the possibility of time-periodic patterns to model several dynamical effects in the brain. From the viewpoint of neurological disorders as well as multi-stable visual perception effects, our results also contribute to a very natural idea: if measurements, such as EEG measurements for epileptic seizures, show a completely different high-oscillation/synchrony regime in comparison to normal brain functioning, one may expect that any underlying mathematical model might 
also undergo a major transition between regimes. In fact, this transition may not only manifest itself in changing model parameters but it may find a clear expression in the mathematical type of the equations themselves. In our work, we have shown that such a structural model transition indeed is possible. For certain parameters, the connectivity kernel may satisfy our assumptions so that typical gradient-flow dynamics is observed leading to energy dissipation and equilibration towards an invariant measure. Yet, for other parameters, our kernel may not lead to a gradient flow and this opens up the possibility of wide variety of neural activity patterns.

\section{Appendix A. Cylindrical Wiener processes in Hilbert spaces}

Let $\left\{v_{i}\right\}_{i \in \mathbb{N}} \subset H$ be a complete orthonormal system for $H$ and let $\left\{\beta_{t}^{i}\right\}_{i \in \mathbb{N}}$ be a collection of independent real-valued standard Brownian motions modeled on a filtered normal probability space $\left(\Omega, \mathcal{F},\left\{\mathcal{F}_{t}\right\}_{t \geq 0}, \mathbb{P}\right)$. Then the cylindrical Wiener process $\left\{W_{t}\right\}_{t \geq 0}$ with covariance $Q=\mathrm{Id}$ has the formal representation

$$
W_{t}=\sum_{i=1}^{\infty} v_{i} \beta_{t}^{i}, \quad t \geq 0,
$$

which is a standard Wiener process in a weaker separable Hilbert space $U$, such that there exists a Hilbert-Schmidt embedding $\iota: H \rightarrow U, \iota \in L_{2}(H, U)$ and $\left\{W_{t}\right\}_{t>0}$ has the covariance operator $\iota \iota^{*}$. By [21, Proposition 4.7 and Proposition 4.8], then $\left(\iota \iota^{*}\right)^{\frac{1}{2}}(U)=H$, and one can always find $U$ and $\iota$ with the above properties such that the representation (A.1) holds; see 21, Chapter 4] for details. For our purposes, it is sufficient to set $U$ equal to the abstract completion of $L^{2}(\mathcal{B})$ with respect to the alternative scalar product $(u, v)_{U}:=\sum_{n=1}^{\infty} n^{-2} \hat{u}_{n} \hat{v}_{n}$, where $u, v \in H$ and $\left\{\hat{u}_{n}\right\},\left\{\hat{v}_{n}\right\} \in \ell^{2}$ are such that $u=\sum_{n=1}^{\infty} \hat{u}_{n} v_{n}$ and $v=\sum_{n=1}^{\infty} \hat{v}_{n} v_{n}$. Then $\iota:=\operatorname{Id}$ is a Hilbert-Schmidt embedding from $H$ into $U$.

\section{REFERENCES}

[1] F. Achleitner and C. Kuehn. On bounded positive stationary solutions for a nonlocal FisherKPP equation. Nonlinear Anal. Theory Methods Appl., 112:15-29, 2015.

[2] S. Amari. Dynamics of pattern formation in lateral-inhibition type neural fields. Biol. Cybern., 27:77-87, 1977.

[3] L. Ambrosio, N. Gigli, and G. Savaré. Gradient flows: In metric spaces and in the space of probability measures. Birkhäuser, 2006.

[4] C. Bachmair and E. Schöll. Nonlocal control of pulse propagation in excitable media. Eur. Phys. J. B, 87(11):276, 2014.

[5] V. Barbu and G. Da Prato. Ergodicity for nonlinear stochastic equations in variational formulation. Appl. Math. Optim., 53(2):121-139, 2006.

[6] F. Barret. Sharp asymptotics of metastable transition times for one-dimensional SPDEs. Ann. Inst. Henri Poincaré Probab. Stat., 51(1):129-166, 2015.

[7] H. Berestycki, G. Nadin, B. Perthame, and L. Ryzhik. The non-local Fisher-KPP equation: travelling waves and steady states. Nonlinearity, 22:2813-2844, 2009.

[8] N. Berglund. Kramers' law: validity, derivations and generalisations. Markov Processes Relat. Fields, 19(3):459-490, 2013.

[9] N. Berglund and B. Gentz. Sharp estimates for metastable lifetimes in parabolic SPDEs: Kramers' law and beyond. Electron. J. Probab., 18(24):1-58, 2013.

[10] N. Berglund, G. D. Gesù, and H. Weber. An Eyring-Kramers law for the stochastic AllenCahn equation in dimension two. Electron. J. Probab., 22:1-27, 2017.

[11] V. I. Bogachev. Gaussian measures. Amer. Math. Soc., Rhode Island, 1998. 
[12] P. C. Bressloff. Stochastic neural field theory and the system-size expansion. SIAM J. Appl. Math., 70(5):1488-1521, 2009.

[13] P. C. Bressloff. Spatiotemporal dynamics of continuum neural fields. J. Phys. A: Math. Theor., 45:(033001), 2012.

[14] P. C. Bressloff. Stochastic neural field theory. In S. Coombes, P. beim Graben, R. Potthast, and J. Wright, editors, Neural fields, theory and applications. Springer-Verlag, Berlin, Heidelberg, 2014.

[15] P. C. Bressloff and M. A. Webber. Front propagation in stochastic neural fields. SIAM J. Appl. Dyn. Syst., 11(2):708-740, 2012.

[16] S. Coombes. Waves, bumps, and patterns in neural field theories. Biol. Cybern., 93:91-108, 2005.

[17] S. Coombes, P. beim Graben, and R. Potthast. Tutorial on neural field theory. In S. Coombes, P. beim Graben, R. Potthast, and J. Wright, editors, Neural fields, theory and applications. Springer-Verlag, Berlin, Heidelberg, 2014.

[18] H. Crauel and F. Flandoli. Attractors for random dynamical systems. Probab. Theory Relat. Fields, 100(3):365-393, 1994.

[19] G. Da Prato and J. Zabczyk. A note on semilinear stochastic equations. Differ. Integral Equations, 1(2):143-155, 1988.

[20] G. Da Prato and J. Zabczyk. Ergodicity for infinite dimensional systems, volume 229 of London Mathematical Society lecture note series. Cambridge Univ. Press, 1996.

[21] G. Da Prato and J. Zabczyk. Stochastic equations in infinite dimensions, volume 152 of Encyclopedia of mathematics and its applications. Cambridge University Press, 2nd edition, 2014.

[22] S. H. da Silva and A. L. Pereira. A gradient flow generated by a nonlocal model of a neural field in an unbounded domain. Topol. Methods Nonlinear Anal., 51(2):583-598, 2018.

[23] M. Enculescu and M. Bestehorn. Liapunov functional for a delayed integro-differential equation model of a neural field. Eur. Phys. Lett., 77:68007, 2007.

[24] G. B. Ermentrout. Neural networks as spatio-temporal pattern-forming systems. Rep. Prog. Phys., 61(4):353, 1998.

[25] G. B. Ermentrout, S. E. Foilas, and Z. P. Kilpatrick. Spatiotemporal pattern formation in neural fields with linear adaptation. In S. Coombes, P. beim Graben, R. Potthast, and J. Wright, editors, Neural fields, theory and applications. Springer-Verlag, Berlin, Heidelberg, 2014.

[26] A. Es-Sarhir and W. Stannat. Invariant measures for semilinear SPDE's with local Lipschitz drift coefficients and applications. J. Evol. Equations, 8(1):129-154, 2008.

[27] A. Es-Sarhir, M. Scheutzow, J. M. Tölle, and O. van Gaans. Invariant measures for monotone SPDEs with multiplicative noise term. Appl. Math. Optim., 68(2):275-287, 2013.

[28] O. Faugeras and J. Inglis. Stochastic neural field equations: a rigorous footing. J. Math. Biol., 71(2):259-300, 2015.

[29] J. C. Ferreira and V. A. Menegatto. Eigenvalues of integral operators defined by smooth positive definite kernels. Integral Equations Oper. Theory, 64(1):61-81, 2009.

[30] J. C. Ferreira and V. A. Menegatto. Positive definiteness, reproducing kernel Hilbert spaces and beyond. Ann. Funct. Anal., 4(1):64-88, 2013.

[31] J. C. Ferreira, V. A. Menegatto, and C. P. Oliveira. On the nuclearity of integral operators. Positivity, 13(3):519-541, 2008.

[32] B. Gess and J. M. Tölle. Multi-valued, singular stochastic evolution inclusions. J. Math. Pures Appl., 101(6):789-827, 2014.

[33] S. A. Gourley. Travelling front solutions of a nonlocal Fisher equation. J. Math. Biol., 41 (3):272-284, 2000. 
[34] R. Hagen, S. Roch, and B. Silbermann. $C^{*}$-Algebras and Numerical Analysis. Marcel Dekker, 2001.

[35] M. Hairer and J. C. Mattingly. Ergodicity of the 2D Navier-Stokes equations with degenerate stochastic forcing. Ann. Math. (2), 164(3):993-1032, 2006.

[36] M. Hairer and J. C. Mattingly. A Theory of Hypoellipticity and Unique Ergodicity for Semilinear Stochastic PDEs. Electron. J. Probab., 16(0), 2011.

[37] J. Inglis and J. MacLaurin. A general framework for stochastic traveling waves and patterns, with application to neural field equations. SIAM J. Appl. Dyn. Syst., 15(1):195-234, 2016.

[38] R. Jordan, D. Kinderlehrer, and F. Otto. The variational formulation of the Fokker-Planck equation. SIAM J. Math. Anal., 29(1):1-17, 1998.

[39] A. Jüngel, C. Kuehn, and L. Trussardi. A meeting point of entropy and bifurcations in cross-diffusion herding. Eur. J. Appl. Math., 28(2):317-356, 2017.

[40] Z. P. Kilpatrick and B. Ermentrout. Wandering bumps in stochastic neural fields. SIAM J. Appl. Dyn. Syst., 12(1):61-94, 2013.

[41] A. N. Kolmogorov. Zur Umkehrbarkeit der statistischen Naturgesetze. Math. Ann., 113: 766-772, 1937.

[42] J. Krüger and W. Stannat. Front propagation in stochastic neural fields: a rigorous mathematical framework. SIAM J. Appl. Dyn. Syst., 13(3):1293-1310, 2014.

[43] J. Krüger and W. Stannat. Well-posedness of the stochastic neural field equation with discontinuous firing rate. J. Evol. Equations, 27(12):1-33, 2017.

[44] C. Kuehn and M. G. Riedler. Large deviations for nonlocal stochastic neural fields. J. Math. Neurosci., 4(1):1-33, 2014.

[45] C. R. Laing. PDE methods for two-dimensional neural fields. In S. Coombes, P. beim Graben, R. Potthast, and J. Wright, editors, Neural fields, theory and applications. SpringerVerlag, Berlin, Heidelberg, 2014.

[46] C. R. Laing and W. C. Troy. PDE methods for nonlocal models. SIAM J. Appl. Dyn. Syst., $2(3): 487-516,2003$.

[47] E. Lang. A multiscale analysis of traveling waves in stochastic neural fields. SIAM J. Appl. Dyn. Syst., 15(3):1581-1614, 2016.

[48] W. Liu and M. Röckner. Stochastic Partial Differential Equations: An Introduction. Universitext. Springer, Cham, 2015.

[49] W. Liu and J. M. Tölle. Existence and uniqueness of invariant measures for stochastic evolution equations with weakly dissipative drifts. Electron. Comm. Probab., 16:447-457, 2011.

[50] R. Marcus. Parabolic Itô equations. Trans. Amer. Math. Soc., 198:177-190, 1974.

[51] R. Marcus. Parabolic Itô equations with monotone nonlinearities. J. Funct. Anal., 29(3): 275-286, 1978.

[52] B. Maslowski. Strong Feller property for semilinear stochastic evolution equations and applications. In Stochastic Systems and Optimization, pages 210-224. Springer, Berlin, Heidelberg, 1989.

[53] A. Mogilner and L. Edelstein-Keshet. A non-local model for a swarm. J. Math. Biol., 38 (6):534-570, 1999.

[54] R. Moreno-Bote, J. Rinzel, and N. Rubin. Noise-induced alternations in an attractor network model of perceptual bistability. J. Neurophysiol., 98(3):1125-1139, 2007.

[55] S. Mück. Semilinear stochastic equations for symmetric diffusions. Stochastics Stochastics Rep., 62(3-4):303-325, 1995.

[56] F. Otto. The geometry of dissipative evolution equations: the porous medium equation. Comm. Partial Differ. Equations, 26(1):101-174, 2001. 
[57] D. Poll and Z. P. Kilpatrick. Stochastic motion of bumps in planar neural fields. SIAM J. Appl. Math., 75(4):1553-1577, 2015.

[58] M. Reed and B. Simon. Methods of modern mathematical physics I. Functional analysis. Academic Press, New York, revised and enlarged edition, 1980.

[59] J. Ren, M. Röckner, and F.-Y. Wang. Stochastic generalized porous media and fast diffusion equations. J. Differ. Equations, 238(1):118-152, 2007.

[60] M. G. Riedler and E. Buckwar. Laws of large numbers and Langevin approximations for stochastic neural field equations. J. Math. Neurosci., 3(1):1, 2013.

[61] M. Röckner and F.-Y. Wang. Non-monotone stochastic generalized porous media equations. J. Differ. Equations, 245(12):3898-3935, 2008.

[62] Z. Sasvári. Multivariate characteristic and correlation functions. De Gruyter, Berlin, Boston, 2013.

[63] T. Schwalger, M. Deger, and W. Gerstner. Towards a theory of cortical columns: From spiking neurons to interacting neural populations of finite size. PLoS Comput. Biol., 13(4): e1005507, 2017.

[64] R. E. Showalter. Monotone operators in Banach space and nonlinear partial differential equations. Mathematical surveys and monographs, Amer. Math. Soc., 1997.

[65] O. Shriki, D. Hansel, and H. Sompolinsky. Rate models for conductance-based cortical neuronal networks. Neural Comput., 15:1809-1841, 2003.

[66] J. Stewart. Positive definite functions and generalizations, an historical survey. Rocky Mt. J. Math., 6(3):409-434, 1976.

[67] C. M. Topaz, A. L. Bertozzi, and M. A. Lewis. A nonlocal continuum model for biological aggregation. Bull. Math. Biol., 68(7):1601, 2006.

[68] J. Touboul, G. Hermann, and O. Faugeras. Noise-induced behaviors in neural mean field dynamics. SIAM J. Appl. Dyn. Syst., 11(1):49-81, 2012.

[69] F. Tricomi. Integral Equations. (Pure and applied mathematics, volume 5). Dover Publications, 1985.

[70] R. van Ee. Dynamics of perceptual bi-stability for stereoscopic slant rivalry and a comparison with grating, house-face, and Necker cube rivalry. Vision Res., 45:29-40, 2005.

[71] R. Veltz and O. Faugeras. Local/global analysis of the stationary solutions of some neural field equations. SIAM J. Appl. Dyn. Syst., 9(3):954-998, 2010.

[72] M. A. Webber and P. C. Bressloff. The effects of noise on binocular rivalry waves: a stochastic neural field model. J. Stat. Mech., 2013:P03001, 2013.

[73] H. Wilson and J. Cowan. A mathematical theory of the functional dynamics of cortical and thalamic nervous tissue. Biol. Cybern., 13(2):55-80, 1973.

[74] S. Xie, A. T. Lawniczak, S. Krishnan, and P. Lio. Wavelet kernel principal component analysis in noisy multiscale data classification. ISRN Comput. Math., 2012:197352, 2012.

[75] J. Zabczyk. Symmetric solutions of semilinear stochastic equations. In Stochastic Partial Differential Equations and Applications II, pages 237-256. Springer, Berlin, Heidelberg, 1989 .

Technical University of Munich, Faculty of Mathematics, Research Unit "Multiscale and StoChastic Dynamics", 85748 Garching bei München, Germany

E-mail address: ckuehn@ma.tum.de

Universität Augsburg, Institut für Mathematik, 86135 Augsburg, Germany

Current address: Universität Ulm, Fakultät für Mathematik und Wirtschaftswissenschaften, Institut für Analysis, 89069 Ulm, Germany

E-mail address: jonas.toelle@math.uni-augsburg.de 